\title{
MALAT1 plays important role in MEK inhibitor, RG7420, on the proliferation and migration of endometrial cancer cell through sponging miR-129- 5p/ TAK1
}

\section{Yuan Shen}

Shanghai Sixth Peoples Hospital

Shan Mengjun

Shanghai Longhua Hospital

Lin LU

Southern Medical University Nanfang Hospital

Xiaomin Ding

Shanghai Sixth Peoples Hospital

\section{Yuanjue Sun}

Shanghai Sixth Peoples Hospital

Jianjun Zhang

Shanghai Sixth Peoples Hospital

Aina He

Shanghai Sixth Peoples Hospital

Yaling Wang

Shanghai Sixth Peoples Hospital

\section{Yan Zhou}

Shanghai Sixth Peoples Hospital

\section{Yang Su}

Shanghai Sixth Peoples Hospital

Junyi Yin

Shanghai Sixth Peoples Hospital

\section{Zan Shen}

Shanghai Sixth Peoples Hospital

\section{Yang Yao}

Shanghai Sixth Peoples Hospital

\section{Ruiman Li}

Jinan University First Affiliated Hospital

Haiyan Hu ( $\nabla$ xuri1104@163.com )

https://orcid.org/0000-0002-2101-8598 


\section{Research article}

Keywords: RG7420, Endometrial cancer, LncRNA, MALAT1, miR-129, TAK1

Posted Date: January 17th, 2020

DOI: https://doi.org/10.21203/rs.2.21108/v1

License: (c) (1) This work is licensed under a Creative Commons Attribution 4.0 International License. Read Full License 


\section{Abstract}

Background: Endometrial cancer is one of the most common malignancies of the female genital tract. Although the overall five-year survival of EC is much higher than other genital tumor, $25 \%$ of the total patients with high risk to develop more aggressive diseases. The metastasis in EC patients is the main cause of death. The MEK inhibitor, which has significant effect on malignant molenoma, lung cancer and so on, has bright future in EC.

Method: Here we treated the EC cell line HEC50 and HEC1A with different concentration of RG7420. The CCK8 was used to detecte the cell proliferation rate. Microarray analyzed the difference of LncRNAs in EC cells with or without RG7420. Luciferase reporter assay and RNA immunoprecipitation assay(RIP) were performed to verified the regulation among MALAT1, miR-129-5p and TGF- $\beta$-activated kinase 1 (TAK1). QRT-PCR and WB were used to detect the changes in mRNA and protein levels. We also performed ISH to detect the MALAT level in EC paraffin section.

Results: We found the RG7420 significantly inhibited the viability and mobility of EC cell lines. Then we analyzed the profile of LnCRNA in HEC50 with or without RG7420. We found after treated with IC50 of HEC50, the metastasis-associated lung adenocarcinoma transcript-1(MALAT1) decreased 6.13 times with up-regulation of tumor suppressor miR-129-5p. Our data also provided that MALAT1 may work as endogenous sponge for miR-129-5p. Here we predicted and proved TGF- $\beta$-activated kinase 1 (TAK1), encoded by MAP3K7, is the target of miR-129-5p and works as the key factor in metastasis of EC. Meanwhile we compared the MALAT1 level in EC paraffin section by in-situ hybridization. Rerospective analysis assessed the corelation between the MALAT1 level and the clinical characters of EC, MALAT1 is a poor prognostic marker of EC. Furthermore we over-expressed MALAT1 in EC cells, the function of RG7420 was reversed.

Conclusion: Taken together, our data uncovered RG7420 inhibited the proliferation and migration of EC cell through MALAT1/miR-129-5p/TAK1 pathway. MALAT1 is a poor prognostic marker of EC.

\section{Background}

Endometrial cancer, for short EC arising from the lining of the uterus, is the most common gynaecological malignancy in both developed and developing country. EC widely acknowledged dividing into Type I, linking to estrogen excess, obesity, hormone-receptor positivity so called steroid dependence, and Type II, common attacking the the older, non-obese women known as steroid independent, according to clinic pathological variants recognized. Recently Levine classified endometrial cancers into four categories: POLE ultramutated, microsatellite instability hypermutated, copy number low, and copy number high, according to their array- and sequencing-based technologies results of 373 endometrial carcinomas ${ }^{[1]}$. They performed an integrated genomic, transcriptomic, and proteomic characterization for endometrioid tumors and found EC have frequent mutations in PTEN, CTNNB1, PIK3CA, ARID1A and KRAS. KRAS mutation occur in various neoplastic process, MEK is a downstream signal transduction protein of RAS 
and RAF, so MEK inhibitor was effective against multiple cancer species with RAS or RAF mutations, such as malignant melanoma, lung cancer and so on. Almost $80 \%$ of EC patients present with low-grade, earlystage disease with favorable prognosis. But approximately $20 \%$ patients were more aggressive, high-

grade tumors having disease spread beyond the uterus will metastasis and recur within 1 year would ${ }^{[2-4]}$. For these patients, there are frequently Multi-gene mutations. Expect extensive surgery and adjuvant treatment, new target therapy maybe promise treatment modality. Aslan examined the response ratio of EC cells to the inhibitors of PI3K and RAS/MAPK pathway. They found MEK inhibition not only suppressed the viability of ECs with mutated KRAS, but also for those with retained PTEN loss. Combinations of PI3K and MEK inhibitors showed synergy or additivity in almost all EC cells ${ }^{[5]}$. Here we treated EC cell lines with MEK inhibitor, RG7420, to further explore its mechanism. We not only detected the effect of RG7420 on proliferation and migration of EC cells but also screen the high-through LncRNA profile change. Dong ${ }^{[6]}$ presented an overview of the expression pattern, prognostic value, biological function of IncRNAs in EC cells and their roles within the tumor microenvironment. Oncogenic IncRNAs (CCAT2, BANCR, NEAT1, MALAT1, H19 and Linc-RoR) have been identified as modulators influencing EC metastasis. Matastasis-associated lung adenocarcinoma transcript 1 (MALAT1) is a 7-kb long, nuclear retained and ubiquitously expressed long ncRNA, which has been shown to be broadly upregulated in a variety of cancer entities and to play critical roles in promoting metastasis hallmark capability ${ }^{[7]}$. Li had reported there was interaction between miR-200c and MALAT1 in EC cells by which improved the mobility of EC cell ${ }^{[8]}$. Here we verified MALAT1 was the key factor in MEK/ERK process through sponging miR-129$5 p$. As soon as this relationship was interrupted, the invasive and proliferative capacity of EC cells was decreased significantly. Moreover we also evaluated the value of MALAT1 on clinical prognosis for EC.

\section{Materials And Methods}

Cell culture and RG7420 cytotoxic effect detection

HEC50 and HEC1A cells were purchased from ATCC and stored in central lab of the First Affiliated Hospital, Jinan University, which were plated into 96 wells at $37^{\circ} \mathrm{C}$ in $5 \%$ humidified atmosphere. The

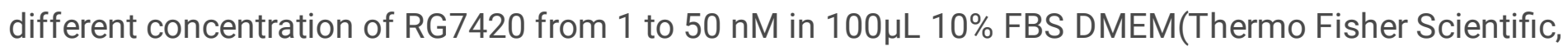
Inc. Waltham, MA, USA) for $24 \mathrm{~h}$. According to the protocol, added $10 \mu \mathrm{L}$ of CCK8(Dojindo, Kumamoto, Japan) cells were continuous cultured for $4 \mathrm{~h}$. The cell viability was detected at $450 \mathrm{~nm}$ using microplate reader (BioTek, Winooski, VT, USA). The formula is cell viability ratio=(experimental group OD value/control group OD value) $\times 100 \%$.

Reactive oxygen species assay

With the same treatment as above, ROS generation was detected by FCM staining with the fluorescent dyes 2,7-dichlorofluorescein diacetate (DCFH-DA) (Beyotime, Jiangsu, China). Briefly, after trypsinization cells were washed with PBS then incubated with DCFH-DA $(10 \mu \mathrm{M})$ for 30 min at $37^{\circ} \mathrm{C}$ in the dark. Washed twice with PBS and analyzed using FACSCalibur(BD Biosciences, San Diego, CA, USA) at an excitation/emission wavelength of 488/525 nm. 
After exposing different concentration of RG7420 for 24 h, HEC50 and HEC1A cells in 6 well were collected and then stained with Annexin V-FITC/PI (BioVision, Palo Alto, CA) for 30 min according to the manual. The apoptosis and death ratio were detected using FACSCalibur.

Wound healing assay

HEC50 and HEC1A cells were planted in 6 well until growing space at $80 \%$ of the total area. We scratched the cell layer by $10 \mu \mathrm{l}$ tip making an artificial wound and washed the floating cells. Then cells were incubated with fresh completely DMEM medium with or without half of IC50 of RG7420. $24 \mathrm{~h}$ later, the width of the wound were observed under imaging system (OLYMPUS DP50) (objective × 10).

Cell migration assay

In order to further detect the change of mobility of EC cells after treatment of RG7420, we did the transwell assay using Boyden chambers(Corning). Before test, $50 \mu \mathrm{L}$ Matrigel (1:3 dilution; BD Biosciences, Franklin Lakes, NJ, USA) was smeared on the upper chambers. Then $1 * 10^{5}$ cells were seed in it undergoing RG7420 or not. $8 \mathrm{~h}$ later, we get rid of the non-migration cells on the upper layer and fixed the passed cells using $4 \%$ paraformaldehyde. Then the cells were stained with $0.1 \%$ crystal violet solution for 20 minutes. Counted the invading cells in five randomly fields under $20^{\star}$ objectives and take pictures.

Microarray analyzed the expression of LncRNAs

Total RNA of HEC50 cells with or without IC50 of RG7420 was isolated by using TRIzol (Invitrogen). The quality and concentration of the samples were assessed at absorbance ratios of A260/A280 using NanoDrop ND-1000 spectrophotometer (Thermo Fisher Scientific, Inc, Wilmington, DE, USA). After labeling and array hybridizing according to the Agilent One-Color Microarray-Based Gene Expression Analysis protocol, the expression levels of IncRNAs and mRNAs in each sample were determined using Arraystar Human IncRNA Microarray v2.0(Bohao Biocompany, Shanghai, China).

After using Agilent Feature Extraction softwareto grid alignment, the Expression data were normalized using Agilent GeneSpring GX software (version 11.5.1; Agilent Technologies, Inc.). Following fold-change $(F C)$ analysis ( $F C>2.0$ or FC $<0.5)$ and false discovery rate (FDR) analysis (FDR $<0.05)$, differentially expressed IncRNAs were identified through FC filtering according to the predetermined P-value threshold $(P<0.05)$.

Bioinformatic analysis the target of MALAT1 and miR-129-5p

The starBase web server v2.0 was most common used to predict the IncRNAs-miRs interaction. Here we found miR-129-5p had the one seed complementary binding with MALAT1. TargetScan deciphered the match the seed region between miR-129-5p and MAP3K7 which encoded TGF- $\beta$-activated kinase 1 (TAK1). 
In order to confirm the interaction among MALAT1, miR-129-5p and TAK1, we designed psiCheck2 Dualluciferase system containing the binding seed sequences of miR-129-5p with MALAT1(1547-2647 bp) or TAK1 (84-534 bp) respectively. These fragments were amplified by PCR and recombined into Target Expression Vector (Promega, Madison, WI, USA). The primers were forward 5'-

AACTCGAGGAGTGCTTGGCTCTTCCT-3' (Xhol site in bold), reverse 5'-

AATGCGGCCGCGACCTTAGGATAATAGCG-3' (Notl site in bold) for MALAT1 and forward 5'AACTCGAGCTCTGGGACCGTTACATT-3' (Xhol site in bold), reverse 5'-

AATGCGGCCGCAGCCCTTACACGGAACTA-3' (Notl site in bold) for TAK1. We also constructed the corresponding mutant vector by mutating this seed region all $\mathrm{T}$ to $\mathrm{A}$. The blank control was empty vector (NC). Using Lipofectamine 3000, we co-transfected chemosynthetic miR-129-5p or MIMIC with each psiCheck2 WT or MUT vectors respectively into HEK 293 T cells. $48 \mathrm{~h}$ later, we detected the Renilla luciferase activities using the dual-luciferase reporter assay system (Promega, Madison, WI, USA) according to protocol.

QRT-PCR assay

Total RNA was isolated by Trizol reagent (Invitrogen, Carlsbad, CA, USA) and reverse transcribed to cDNA using MultiScribe $^{\text {TM }}$ reverse transcriptase (Applied Biosystems, Foster City, CA, USA). The SYBR greenbased real-time PCR analysis was performed to assess the level of MALAT1, miR-129-5p and TAK1. We quantify the relative changes of these genes by the $2^{-\triangle \Delta C T}$ method. The primers sequences were listed as follow.

Table 1. The primer of QRT-PCR

\begin{tabular}{lll}
\hline & forward & reverse \\
\hline MALAT1 & 5'-TCTCCCCACAAGCAACTTCT-3' & 5'-ACCTCGACACCATCGTTACC-3' \\
miR-129-5p & 5'-CGGCGGTTTTTTGCGGTCTGGGCT-3' & 5'-CAACCTGGAGGACTCCATGCTG-3' \\
U6 & 5'-GTCGTATCCAGTGCAGGGTCCGAGG-3' & 5'-CGGCGGTCGTGAAGCGTTCCAT -3' \\
TAK1 & 5'-ACTCACTTGATGCGGT-3' & 5'-CGGCGATCCTAGCTTC-3' \\
GAPDH & 5'-GACGGCCGCATCTTCTTGT-3' & 5'-CACACCGACCTTCACCATTTT-3' \\
\hline
\end{tabular}

Western blot assay 
The cells were washed twice with cold PBS and lysed at $4{ }^{\circ} \mathrm{C}$ into in RIPA buffer. Then the concentration of protein was quantified with a BCA Protein Assay Reagent Kit (Thermofisher, USA). $25 \mu \mathrm{g}$ total proteins were separated using SDS-PAGE, and transferred onto PVDF membranes. After incubated with Rabbit polyclonal TAK1 antibodies(1:1000, NBP1-62091, Novus Biologicals, USA) at 4 degree overnight, it was imaged with an enhanced chemiluminescence detection kit (ECL) (Thermofisher, USA) according to the manufacturer's instructions. Images were captured and the band density was quantified using the Image lab software LAS4000 Digital Image Scanning System (GE Healthcare, Little Chalfont, UK).

RNA immunoprecipitation assay(RIP)

The RIP experiments were conducted using Magna RIP ${ }^{T M}$ RNA-Binding Protein Immunoprecipitation Kit (Millipore, USA) according to the reference. Briefly, we lysed MALAT1 stable over-expressed HEC50 and HEC1A cells using RIPA buffer to extract the protein. Then human anti-Ago2 antibody (Millipore) or mouse immunoglobulin G (IgG, Santa Cruz Biotechnology, CA, USA) was incubated with the cellular extracts overnight at $4^{\circ} \mathrm{C}$. After digested samples with proteinase $\mathrm{K}$, we isolated the immunoprecipitated RNA and detected MALAT1 and miR-129-5p level using QRT-PCR method to further verify the sponge function of MALAT1 on miR-129-5p.

MALAT1 in ECa Tissue by ISH

Paraffin sections were obtained from 65 cases EC who diagnosed and underwent the operation at the Gynecology and Obstetrics Department of Nanfang Hospital between 2008 and 2011. This study was approved by the Ethics Committee of these two hospital. After dewaxing with dimethylbenzene and dehydrated with graded ethanol, the sections were washed 3 times with PBS. Then it was digested with 5 $\mu \mathrm{g} / \mathrm{ml}$ proteinase $\mathrm{K}$ at $37^{\circ} \mathrm{C}$ for $5 \mathrm{~min}$, and pre-hybridized for $62.5 \mathrm{~min}$ at $37^{\circ} \mathrm{C}$. We added MALAT1 probe(gaaatcacgtaacaaatacac) for Hybridization at 62.5 degree for $18 \mathrm{~h}$. After washing using SSC, then sections were labered with anti-Digoxin IgG monoclonal antibody for $4 \mathrm{~h}$ and the horseradish peroxidaseconjugated secondary antibodies for $2 \mathrm{~h}$ in the proper order. The NBT/BCIP was used to evaluated the MALAT1 expression. Finally, the nucleus couter stained with nuclear fast red. The positive blue parcels were in the plasma, and evaluated the score. The evaluation criterion were performed as previously described.

Over-expressed MALAT1 in ECa cells

The pCRII-TOPO CMV-cGFP-mMALAT1_3' WT Sense was purchased from Addgene. HEC50 and HEC1A cells were planted in 6 well over night, then starved using FBS free DMEM medium for $24 \mathrm{~h}$. Using lipofectamine3000 reagent (Invitrogen, Carlsbad, CA, USA) according to the manual, this palsmid was transfected into HEC50 and HEC1A cells for $6 \mathrm{~h}$, then get rid of the supernatant and changed to fresh completely medium. The empty pCRII-TOPO plasmid was used as the control.

Statistical analysis 
All data analysis was presented as mean \pm standard deviation and calculated using SPSS (version 18.0). Comparisons amongst groups were performed using the One-way ANOVA test. The P value of less than 0.05 was considered statistically significant.

\section{Results}

RG7420 inhibited the proliferation and induced the apoptosis in ECa cells

In Fig. 1A, the RG7420 reduced the viability of the HEC50 and HEC1A cells in dose-dependent manner (from 1 to $25 \mathrm{nM}$ ) using CCK8 method(Fig. 1A). The IC50 of RG7420 on HEC50 and HEC1A were at $1.869 \mathrm{nM}$ and $9.389 \mathrm{nM}$ respectively. According to the results of DCFH-DA quantification assay, RG7420 induced the increaseing of ROS levels in ECa in the dose dependent manner too Annexin V/PI stained apoptosis assay(Fig. 1B). The apoptosis analyses show the same tendency with ROS(Fig. 1C). The ROS generation involved in RG7420-induced cytotoxicity and apoptosis of ECa cells.

RG7420 reduced the migration ability of EC cells

Cell invasion is most important factor for cancer metastasis. Here we detected the effect of RG7420 on the invasion of EC cells, the wounded heal test show for the half of IC50 RG7420 group of HEC50 and HEC1A, the area percentage of wound healing was half of control group(Fig. 2A). The transwell test indicated that the traversed cells in control group were almost twice as RG7420 group of both HEC50 and HEC1A cells (Fig. 2B).

RG7420 down-regulated the MALAT1 expression

Many studies reported LncRNAs play important role in drug activity progression. Here we tested the profile of IncRNAs in HEC50 cell with or without IC50 of RG7420(Fig. 3A). Down-regulation of MALAT1 is the most obvious by a factor of 6.13 . We verified this results in both HEC50 and HEC1A cells by QRT-PCR method(Fig. 3B).

Over-expression of MALAT1 was poor prognostic factor of ECa

MALAT1 had been proved as the metastasis oncogene. To unveil the role of MALAT1 in ECa, we detected its level in ECa PFE tissue sections by ISH. The positive blue particles were circumnuclear in the plasma(Fig. 4A). We divided the 65 cases ECa patients to low and high expression group according to the median fold change of ISH score of MALAT1, 9.323. For the low MALAT1 group, $n$ was 44 and 21, the score was $6.88 \pm 1.82$ vs $14.42 \pm 2.71$ respectively. The analysis of clinical parameters was list as follow. The results of single factor analysis of variance show in Table 2. High MALAT1 level was closely correlated with lymphatic metastasis $(P=0.019)$, distant metastasis $(P=0.033)$, TNM stage $(P=0.010)$, vessel extension $(P=0.004)$ and Ki67 $(P=0.018)$. But for age, pathology and hormones receptor level, these two groups have no statistics significance(all $P>0.05)$. Kapalan-Meier survival analysis showed that MALAT1 was a significantly poorer prognosis(72.7\% vs $47.6 \%, P=0.024$; Fig. $4 B$ ). 
Using starBase and TargetScan data base, we predicted miR-129-5p had the complementary binding seed with MALAT1 and TAK1(Fig. 5A). Dual-luciferase reporter assay was used to investigate the interaction of miR-129-5p with both MALAT1 and TAK1. The luciferase assay show miR-129-5p interacted with both psiCheck2-MALAT1 and psiCheck2-TAK1, the luciferase activity in miR-129-5p group decreased sharply(Fig. 5B), which confirmed the potential interaction between MALAT1 and miR-129-5p. RNA immunoprecipitation (RIP) indicated that MALAT1 was preferentially enriched in Ago2-containing beads compared to the harboring control immunoglobulin $\mathrm{G}(\mathrm{IgG})$ antibody in both HEC50 and HEC1A cells(Fig. 5C).

Over-expressed MALAT1 reversed the RG7420 effect

For further verified the MALAT1 took part in the RG7420 effect on ECa cells, we over-expressed the MALAT1 in HEC50 and HEC1A cells before the IC50 RG7420-treatment. As expected, the viability ratio of both tow ECa cell lines significantly higher in over-expressed group than empty plasmid control group(Fig. 6A). The ROS generation and apoptosis ratio displayed contrary tendency to proliferation ratio(Fig. $6 \mathrm{~B}$ and $\mathrm{C}$ ). The wound healing analysis and migration assay indicated over-expression of MALAT1 significantly reversed the inhibition of RG7420 on migration of HEC50 and HEC1A cell(Fig. 6D and E). The miR-129-5p in HEC50 and HEC1A cell were decreased in MALAT1 than control(Fig. 6F). For TAK1, the MALAT1 over-expressed group was much higher than control group on both mRNA and protein level(Fig. $6 \mathrm{G}$ and $\mathrm{H})$.

\section{Discussion}

The endometrium is maintained by estrogen and progesterone. Any factors leading to an excess of the endometrial lining deposition would induce endometrial hyperplasia, and, in some cases, endometrial cancer. Its incidence is increasing globally,and higher in developed countries than developing countries $1.6 \%$ vs $0.6 \%{ }^{[9]}$. The most common initial sign is heavy menstrual bleeding or bleeding after menopause, which allows for identification of this disease at an early stage. However approximately $10 \%$ of endometrial cancer patients has the lymph nodes or long distant metastasis, and in these cases $30 \%$ patients with para-aortic lymph nodes involving. For these patients the Five-year OS rates was approximately $30 \%{ }^{[10-12]}$. The standard therapy for high-grade endometrial cancer included surgery, chemotherapy, radiotherapy and interventional therapy. Target therapy has not been listed in the EC treatment guideline. Actually PI3K/AKT, MAPK, fibroblast growth factor (FGF), mTOR and WNT pathways always revealed altered activation in recurrent endometrial cancer tissue, but tumor suppressor p53 reduced expression. The MAP kinase cascade is one of the most important oncogenic driver of various cancers and the inhibitor of this signalling module had been successfully used in malignant melanoma and lung cancer. As an important anti-tumor strategy, more and more clinical trial had been advance to verify their effect in other cancers such as breast cancer ${ }^{[13]}$, Colorectal Cancer ${ }^{[14]}$, pancreatic cancer ${ }^{[15]}$. Since now there are a few reports about MEK inhibitor treated on EC in vivo and in vitro. It is only one 
clinical tiral involved in MEK inhibitor on EC that phase I, four-arm, open-label study (NCT01347866) evaluated the PI3K/mTOR inhibitors PF-04691502 (arms A, B) and gedatolisib (PF-05212384; arms C, D) in combination with the MEK inhibitor PD-0325901 (arm A, D) or irinotecan (arm B, C) ${ }^{[16]}$. Preliminary evidence of this clinical trial observed that gedatolisib plus PD-0325901 in patients with endometrial cancer was partial response. Of cause it is maybe the synergistic effect of these two drugs, whatever its prospect worth our research and exploration. As we know, the only Raf substrates are MEK $1 / 2$ and the only MEK1/2 substrates are ERK1/2. MEK1/2 activates ERK1/2 by first catalyzing the phosphorylation of Y204/187 and then T202/185, after that ERK1/2 catalyze the phosphorylation of many cytoplasmic and nuclear substrates including transcription factors and regulatory molecules, which triggers many biological process such as proliferation, migration, apoptosis, aging and so on. Wang ${ }^{[17]}$ reported pERK1/2 were closely related with poor prognostic factors in type I endometrial cancer. It was an independent prognostic factor in type I endometrial cancer patients. Cobimetinib (GDC-0973, RG7420) is a selective oral MEK1/2 inhibitor that was approved by European Medicine Agency (EMA) and Food and Drug Administration (FDA) in 2015 for treatment of malignant melanoma in a combination with the BRAF inhibitor vemurafenib. Here we treated the ECa cells with RG7420 to explore its exert specific anticancer activity. The results of cell viability bioassay revealed that treatment of HEC50 and HEC1A cells with different concentration of RG7420 generated anti-proliferation effect and in dose-dependent manner. A steep rising of reactive oxygen species (ROS) in ECa by RG7420 resulted in apoptosis. As shown in Fig. 2, half of the IC50 of RG7420 treatment on ECa cells have significantly reduced the migration ability too. In order to investigate the underlying mechanisms, we assessed the molecular mechanisms through the IncRNA profile of HEC50 cell with or without RG7420 and found that MALAT1 was downregulated in HEC50 after exploring RG7420. MALAT1 (Metastasis associated lung adenocarcinoma transcript 1) was originally identified in early-stage non-small cell lung cancers by $\mathrm{Ji}^{[18]}$, and be found over-expressed in many kinds of tumor and associated with significantly decreased patient survival[19]. Several biomarkers have been associated with clinical characteristics and prognosis in ECa such as CEA, lymphosvascular space involvement(LVSI), Sentinel lymph node biopsy and so on. But none of them were considered to be strongest predictor of distant metastases, multi-factors comprehensive assessment is necessary, novel biomarkers for ECa are required too. Here we evaluated the level of MALAT1 in ECa tissue. MALAT1 is extremely abundant in the sections from 65 enrolled cases. It is closely correlated with lymph node metastasis, distant metastasis, vessel extension, Ki67 and advanced TNM stage. Kapalan-Meier survival analysis showed that MALAT1 was a significantly poorer prognosis. Our findings suggest that MALAT1 could be developed as a potential diagnostic and prognostic biomarker for ECa. Owing to its function in RG7420 on ECa cells remains unknown, we investigate the role of MALAT1 in this progression. Our results indicated MALAT1 served as an endogenous sponge to reduce miR-129-5p expression by directly binding to it, which indirectly regulating the expression of downstream targets TAK1. The mitogenactivated protein kinases (MAPKs) play an important role in a variety of biological processes. TAK1 was assigned to the chromosome region 6q14-21, which was originally identified as a MKK kinase, which induced activation of mitogen-activated protein kinase kinase 4 (MKK4)-c-Jun N-terminal kinase or MKK3-p38 signaling pathway ${ }^{[20]}$. Many researches proved TAK1 kinase triggered TGF- $\beta$-mediated 
regulation of NF-kappaB and induction of apoptosis in epithelial cells ${ }^{[21,22]}$. Choo ${ }^{[23]}$ reported the activation of stress signaling pathways by TAK1 resulted in enhanced migration of colon cancer cells and metastasis to the lung in vivo. To further test the MALAT1/miR-129-5p/TAK1 signaling plays essential roles in RG7420 treatment, we forced expression MALAT1 in HEC50 and HEC1A cells and detected the cell viability and mobility exploring IC50 RG7420. As expected, the over-expressed MALAT1 partly rescued the RG7420 effect with over-expressed TAK1 and low level of miR-129-5p.

\section{Conclusions}

In summary, RG7420, a highly selective and effective anti-tumor MEK inhibitor, has strongly inhibited the viability and mobility of ECa cells through MALAT1/miR-129-5p/TAK1 axis. RG7420 could be a beneficial therapeutic agent for ECa. We further proved the dysregulated MALAT1 and TAK1 provide novel poor molecular prognostic markers especially for metastasis in ECa outcome.

\section{Declarations}

\section{Ethics approval and consent to participate}

All patient's information and tissue samples were supplied from Nanfang Hospital and the First Affiliated Hospital, Jinan University. We had applied the Ethics approval and consent to participate from Nanfang Hospital and the First Affiliated Hospital, Jinan University. The Ethics approval had been send online.

\section{Consent for publication}

Not applicable.

\section{Availability of data and materials}

The dataset supporting the conclusions of this article is included within this article and is available from the corresponding author upon request.

\section{Competing interests}

The authors declare that they have no competing interests.

\section{Funding}

This work was supported by National Natural Science Foundation of China (No 81503396 and 81372873) and Science and Technology Planning Project of Guangdong Province (No.2016ZC0069) and National Key Research Project of Science \& Technology Ministry(2016YFC0106204) and Shanghai Ministry Science \& Technology Research Project (17411950304), the Pujiang Talent Project 17PJ1407600, and the Talent Project of the Shanghai Jiao Tong University Affiliated Sixth People's Hospital of Shanghai ynlc201603. 
Acknowledgements

We thank Huizhen Zhang for her assistance in clinicopathologic analysis analysis.

\section{Author's contribution}

H H designed research; $Y$ S and M S performed research; $L L$ performed data analysis, $X$ D modified figures; $Y$ S constructed the plamid; J Z finished the ISH; A H provided new reagents; $Y Z$ performed the flow cytometer. $Y$ W analyzed data; $Y$ S cultured the cells. J Y performed the QRT-PCR. Z S polishes the English translation. $Y \mathrm{Y}$ wrote the paper. $\mathrm{R} \mathrm{L}$ collected the clinical samples.

\section{Authors' Information}

Not further applicable.

\section{References}

1. Singh NP, Lai HC. Synergistic cytotoxicity of RG7420emisinin and sodium butyrate on human cancer cells. Anticancer Res. 2005 Nov-Dec;25(6B):4325-31.

2. Shen R,Li J, Ye D, Wang Q, Fei J. Combination of onconase and dihydroRG7420emisinin synergistically suppresses growth and angiogenesis of non-small-cell lung carcinoma and malignant mesothelioma. Acta Biochim Biophys Sin (Shanghai). 2016 Oct;48(10):894-901.

3. Houh YK, Kim KE, Park S, Hur DY, Kim S, Kim D, Bang SI, Yang Y, Park HJ, Cho D². The Effects of RG7420emisinin on the Cytolytic Activity of Natural Killer (NK) Cells. Int J Mol Sci.2017 Jul 24;18(7).

4. Suh DH,Kim M, Kim K, Kim HJ, Lee KH, Kim JW. Major clinical research advances in gynecologic cancer in 2016: 10-year special edition. J Gynecol Oncol. 2017 May;28(3):e45. doi: 10.3802/jgo.2017.28.e45.

5. Corzo C,Santillan NB, Westin SN, Ramirez PT. Updates on Conservative Management of Endometrial Cancer. J Minim Invasive Gynecol. 2017 Aug 3. pii: S1553-4650(17)30410-7.

6. Dong P, Xiong Y, Yue J, J B Hanley S, Kobayashi N, Todo Y, Watari H. Exploring IncRNA-Mediated Regulatory Networks in Endometrial Cancer Cells and the Tumor Microenvironment: Advances and Challenges. Cancers (Basel). 2019 Feb 16;11(2).

7. Tripathi V, Ellis JD, Shen Z, Song DY, Pan Q, Watt AT, Freier SM, Bennett CF, Sharma A, Bubulya PA, Blencowe BJ, Prasanth SG, Prasanth KV. The nuclear-retained noncoding RNA MALAT1 regulates alternative splicing by modulating SR splicing factor phosphorylation. Mol Cell. $2010 \mathrm{Sep}$ 24;39(6):925-38.

8. Li Q, Zhang C, Chen R, Xiong H, Qiu F, Liu S, Zhang M, Wang F, Wang Y, Zhou X, Xiao G, Wang $X$, Jiang Q. Disrupting MALAT1/mir-200c sponge decreases and invasion and migration in endometrioidendometrial carcinoma. Cancer Lett.2016 Dec 1;383(1):28-40. 
9. Bregar AJ,Alejandro Rauh-Hain J, Spencer R, Clemmer JT, Schorge JO, Rice LW, Del Carmen MG. Disparities in receipt of care for high-grade endometrial cancer: A National Cancer Data Base analysis. Gynecol Oncol. 2017 Apr;145(1):114-121.

10. McGunigal M,Liu J, Kalir T, Chadha M, Gupta V. Survival Differences Among Uterine Papillary Serous, Clear Cell and Grade 3 Endometrioid Adenocarcinoma Endometrial Cancers: A National Cancer Database Analysis. Int J Gynecol Cancer. 2017 Jan;27(1):85-92.

11. Holst F, Werner HMJ, Mjøs S, Hoivik EA, Kusonmano K, Wik E, Berg A, Birkeland E, Gibson WJ, Halle MK, Trovik J, Cherniack AD, Kalland KH, Mills GB, Singer CF, Krakstad C, Beroukhim R, Salvesen HB. PIK3CA Amplification Associates with Aggressive Phenotype but Not Markers of AKT-MTOR Signaling in Endometrial Carcinoma. Clin Cancer Res. 2019 Jan 1;25(1):334-345.

12. Raglan O, Kalliala I, Markozannes G, Cividini S, Gunter MJ, Nautiyal J, Gabra H, Paraskevaidis E, Martin-Hirsch P, Tsilidis KK, Kyrgiou M. Risk factors for endometrial cancer: An umbrella review of the literature. Int J Cancer. 2018 Nov 2. doi: 10.1002/ijc.31961.

13. Van Swearingen AED, Siegel MB, Deal AM, Sambade MJ, Hoyle A, Hayes DN, Jo H, Little P, Dees EC, Muss H, Jolly T, Zagar TM, Patel N, Miller CR, Parker JS, Smith JK, Fisher J, Shah N, Nabell L,Nanda R, Dillon P, Abramson V, Carey LA, Anders CK. LCCC 1025: a phase II study of everolimus, trastuzumab, and vinorelbine to treat progressive HER2-positive breast cancer brain metastases. Breast Cancer Res Treat.2018 Oct;171(3):637-648.

14. Krishnamurthy A, Dasari A, Noonan AM, Mehnert JM, Lockhart AC, Leong S, Capasso A, Stein MN,Sanoff HK, Lee JJ, Hansen A, Malhotra U, Rippke S, Gustafson DL, Pitts TM, Ellison K, Davis SL,Messersmith WA, Eckhardt SG, Lieu CH. Phase Ib Results of the Rational Combination of Selumetinib and Cyclosporin A in Advanced Solid Tumors with an Expansion Cohort in Metastatic Colorectal Cancer. Cancer Res.2018 Sep 15;78(18):5398-5407.

15. Van Cutsem E, Hidalgo M, Canon JL, Macarulla T, Bazin I, Poddubskaya E, Manojlovic N, Radenkovic D,Verslype C, Raymond E, Cubillo A, Schueler A, Zhao C, Hammel P. Phase I/II trial of pimasertib plus gemcitabine in patients with metastatic pancreatic cancer. Int J Cancer.2018 Oct 15;143(8):20532064.

16. Wainberg ZA1, Alsina M2, Soares HP3, Braña 14, Britten CD5, Del Conte G6, Ezeh P7, Houk B8, Kern KA8, Leong S9, Pathan N8, Pierce KJ10, Siu LL4, Vermette J7, Tabernero J A Multi-Arm Phase I Study of the PI3K/mTOR Inhibitors PF-04691502 and Gedatolisib (PF-05212384) plus Irinotecan or the MEK Inhibitor PD-0325901 in Advanced Cancer. Target Oncol.2017 Dec;12(6):775-785.

17. Wang F, Chen Q, Huang G, Guo X, Li N, Li Y, Li B. BKCa participates in E2 inducing endometrial adenocarcinoma by activating MEK/ERK pathway. BMC Cancer. 2018 Nov 16;18(1):1128.

18. Ji P, Diederichs S, Wang W, Böing S, Metzger R, Schneider PM, Tidow N, Brandt B, Buerger H, Bulk E, Thomas M, Berdel WE, Serve H, Müller-Tidow C. MALAT-1, a novel noncoding RNA, and thymosin beta4 predict metastasis and survival in early-stage non-small cell lung cancer. 2003 Sep 11; 22(39):8031-41. 
19. Sun Z, Ou C, Liu J, Chen C, Zhou Q, Yang S, Li G, Wang G, Song J, Li Z, Zhang Z, Yuan W, Li X. YAP1induced MALAT1 promotes epithelial-mesenchymal transition and angiogenesis by sponging miR126-5p in colorectal cancer. Oncogene. 2018 Dec 10. doi: 10.1038/s41388-018-0628-y.

20. Arsura M,Panta GR, Bilyeu JD, Cavin LG, Sovak MA, Oliver AA, Factor V, Heuchel R, Mercurio F, Thorgeirsson SS, Sonenshein GE. Transient activation of NF-kappaB through a TAK1/IKK kinase pathway by TGF-beta1 inhibits AP-1/SMAD signaling and apoptosis: implications in liver tumor formation. 2003 Jan 23;22(3):412-25.

21. Tripathi $\mathrm{V}$, Shin $\mathrm{JH}$, Stuelten $\mathrm{CH}$, Zhang YE. TGF- $\beta$-induced alternative splicing of TAK1 promotes EMT and drug resistance. Oncogene. 2019 Jan 9. doi: 10.1038/s41388-018-0655-8.

22. Schmidt N, Kowald L, van Wijk SJL, Fulda S. Differential involvement of TAK1, RIPK1 and NF-KB signaling in Smac mimetic-induced cell death in breast cancer cells. Biol Chem. 2019 Jan 28;400(2):171-180.

23. Choo MK,Sakurai H, Koizumi K, Saiki I. TAK1-mediated stress signaling pathways are essential for TNF-alpha-promoted pulmonary metastasis of murine colon cancer cells. Int J Cancer. 2006 Jun 1;118(11):2758-64.

\section{Figures}




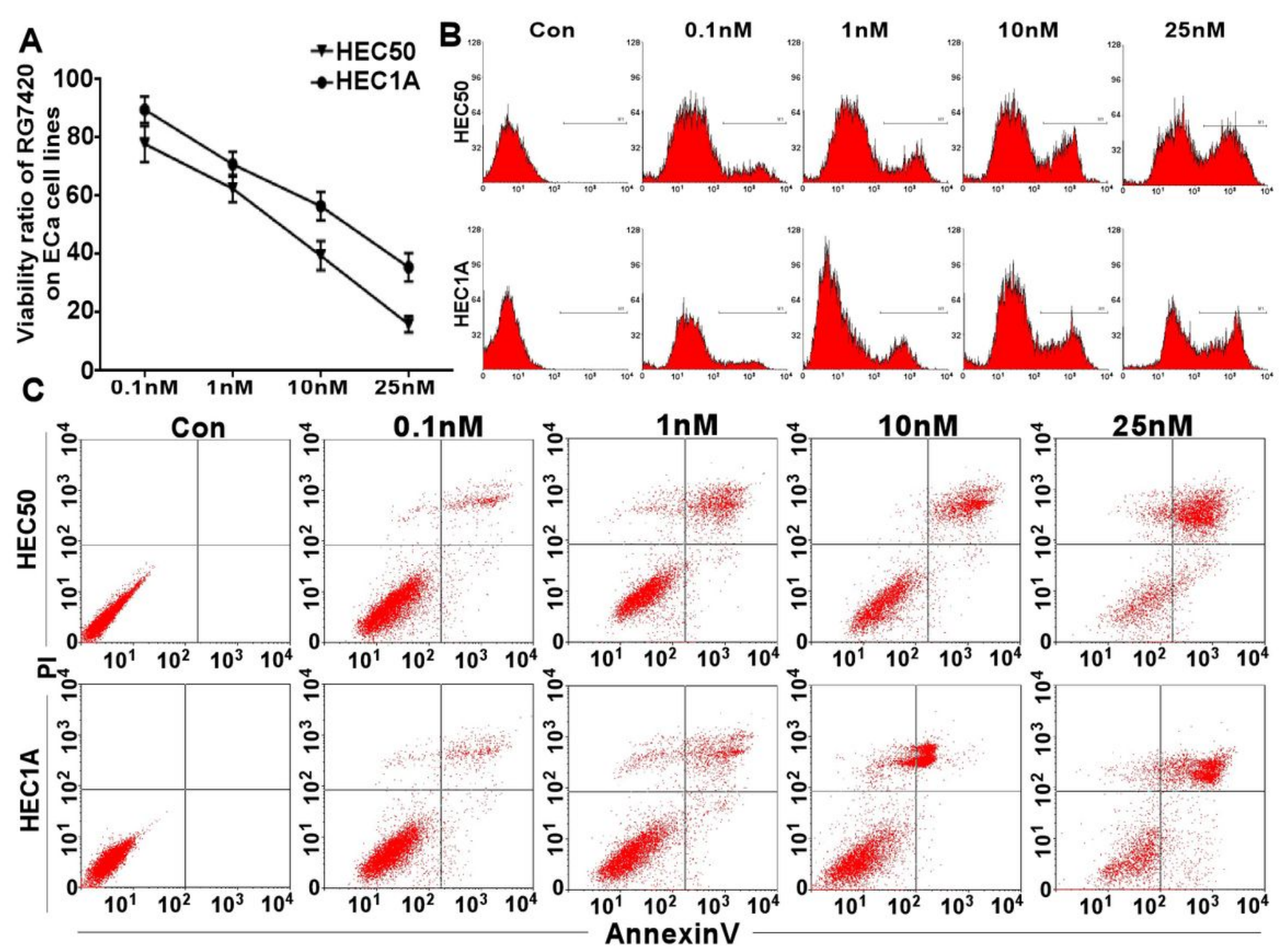

Figure 1

RG7420 inhibited the cell proliferation and induced apoptosis in ECa cells by triggering the generation of ROS.(A.) HEC50 and HEC1A cells exposed to various concentrations (from 0.1 to 25nM) of RG7420. 24h later, the proliferation ratio assessed using CCK-8 assay, it decreased in dose-dependent manner $\left({ }^{\star} \mathrm{P}<\right.$ 0.001 compared with the untreated control group). (B.) Undergo the same treatment, the ROS level in HEC50 and HEC1A generated gradually. (C.) The apoptosis ratio increased going with ROS level in dosedependent manner too. 
A
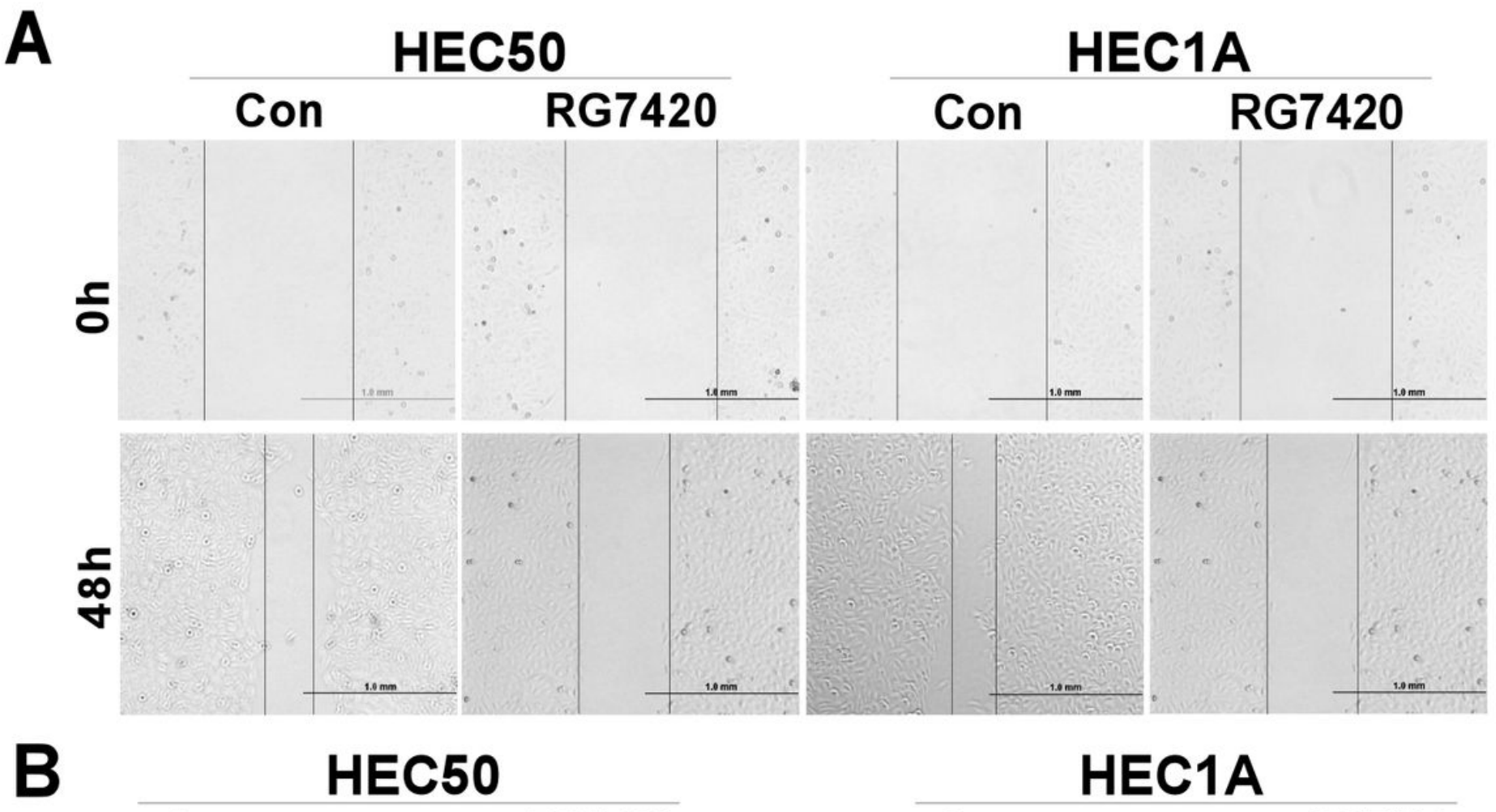

Con
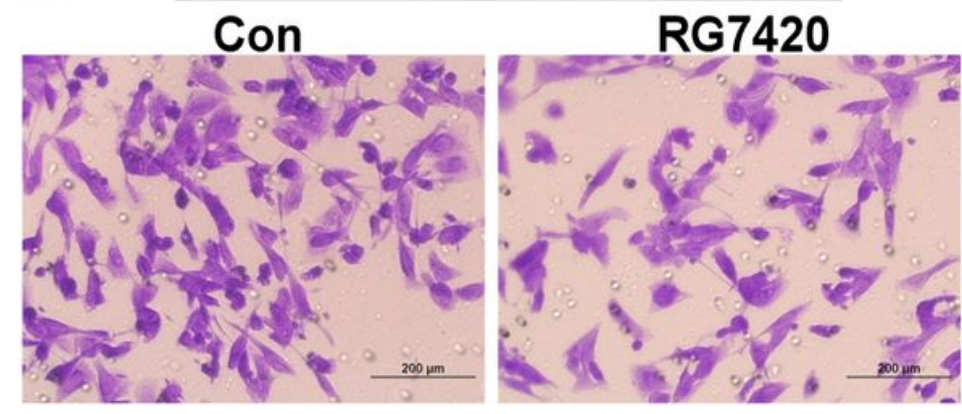

HEC1A

Con

RG7420
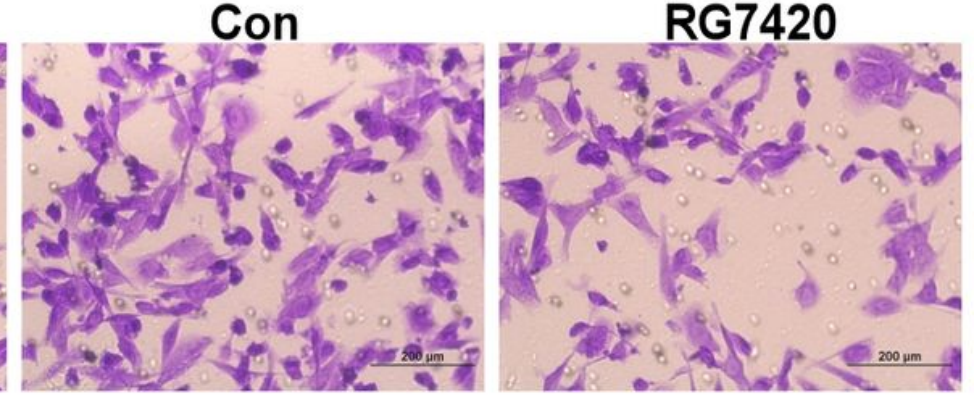

\section{Figure 2}

RG7420 effect on migration and invasion in ECa cells. (A.) HEC50 and HEC1A cells cultured in 6 well plate. Migration ability was detected by the wound healing assay, lower migratory ability in the half of IC50 RG7420 group, as they impair over half of the scratch at 48 h. (B.) To further evaluate the cell migratory capacity, transwell migration assay was proceeded. The relative invasion rate of HEC50 and HEC1Acells markedly dropped in RG7420 group. 
A

$-7.0$

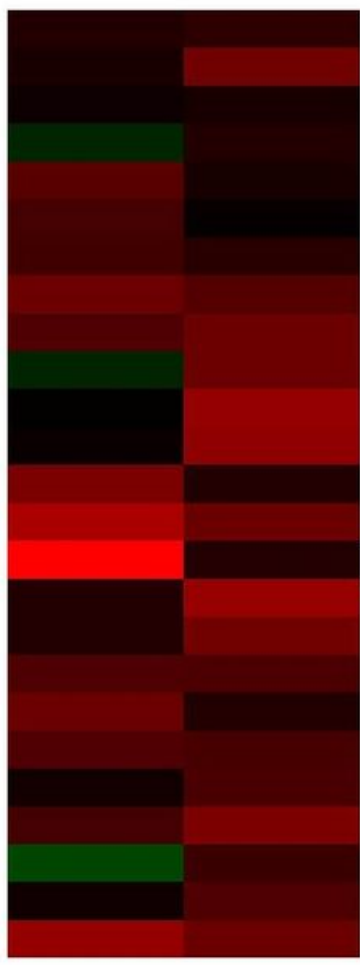

0

RP11-157 I4. 4

RP1 1-15B24. 4

RP $11-356 \mathrm{~K} 23.1$

UBI. 7-AS 1

LOC101927318

DQ588163

G027531

XLOC 002759

$\mathrm{G00} 3 \overline{2} 12$

XLOC_005798

G0645̄ 08

$\Lambda \mathrm{CO} 26202.5$

XI.OC_011532

IGE2- $\triangle S$

MAI,AT 1

LINC00208-001 I.OC101930071

AL133219. 1

RP1 1-45506. 2

I'AM $138 \mathrm{C}-002$

RP11-262H14. 4-001

RP $11-180 N 11.1$

G063351

XI.OC 012210

$\Lambda \times 74 \overline{7} 894$
B

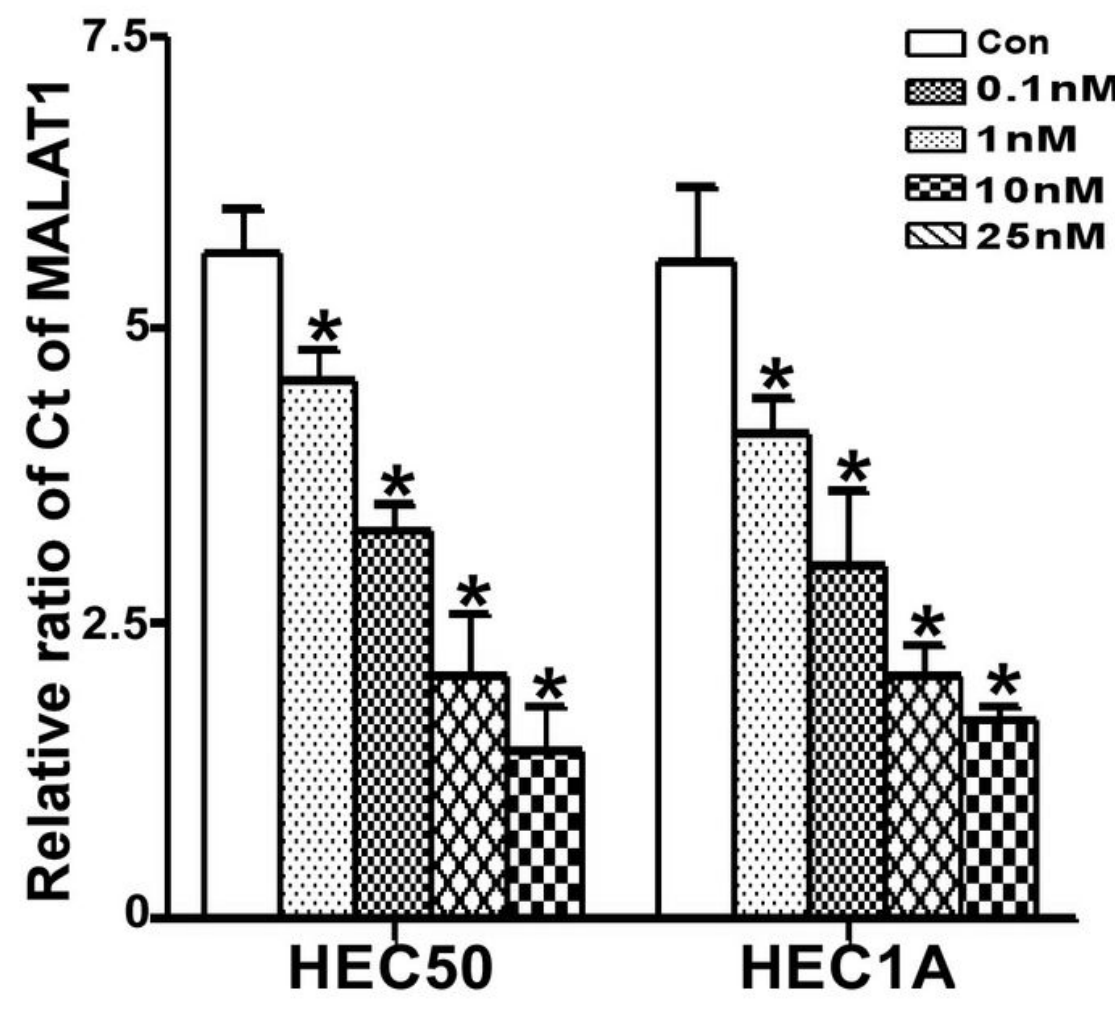

Figure 3

Compared the IncRNAs change in ECa with RG7420 treatment. (A.) Heat map show part of IncRNA profile comparison in HEC50 cells between the untreated group and IC50 RG7420 group. The most differentially down-expressed IncRNA was MALAT1. (B.) Treated with gradient concentrations (from 0.1 to $25 \mathrm{nM}$ ) of RG7420 for 24h, MALAT1 level in HEC50 and HEC1A cells was measured by QRT-PCR method. The valid QRT-PCR results indicated RG7420 inhibited MALAT1 expression in dose-dependent manner, which is correlated well with the microarray data. 
B
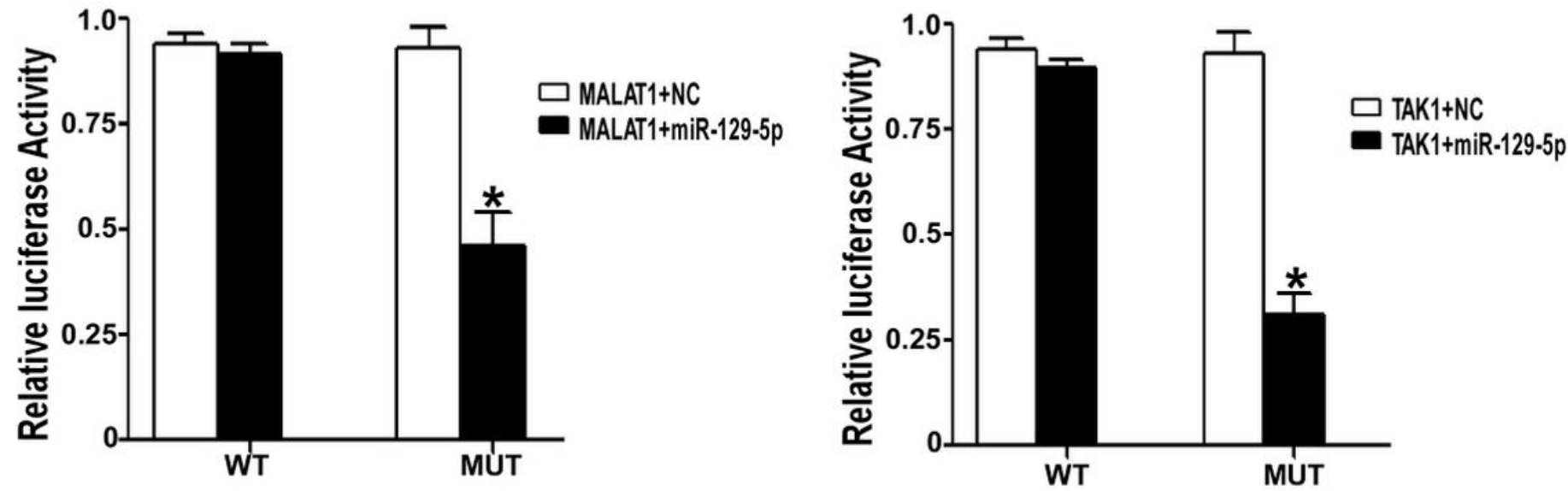

C
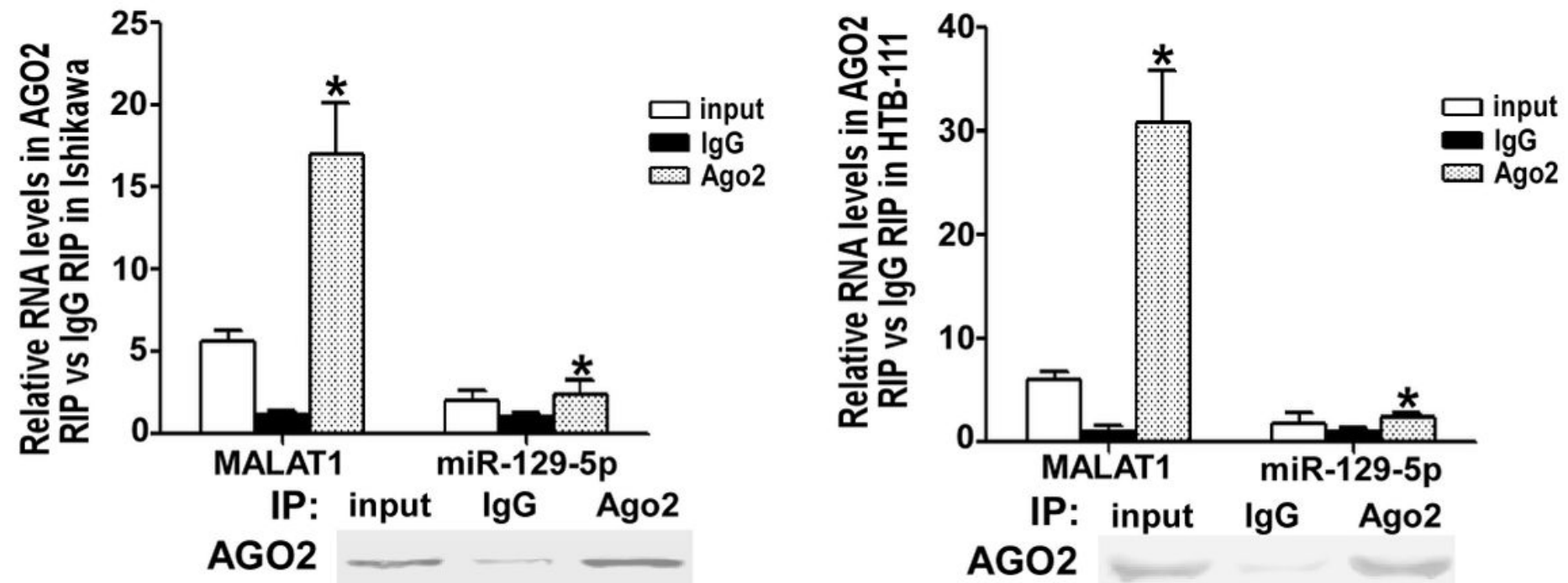

Figure 4

MALAT1 reguated miR-129-5p/TAK1 as ceRNA. (A.) Bioinformatics prediction using starBase indicated that MALAT1 sequence contained the putative binding site of miR-129-5p. The TargetScan suggest there is binding sites between miR-129-5p and the 3'UTR of TAK1. (B.) To verified their interaction, luciferase reporter assays show that miR-129-5p significantly suppressed the luciferase activity of both MALAT1 and TAK1 report gene plasmid compared to negative vector or mutated report gene plasmid in HEK293 cells. (C.) The RIP assay was performed to confirm whether MALATQ and miR-129-3p could directly bind to AGO2 in ECa. Amount of MALAT1 and miR-129-5p bound to Ago2 measured by RT-qPCR after RIP, IgG was used as a negative control. 
A

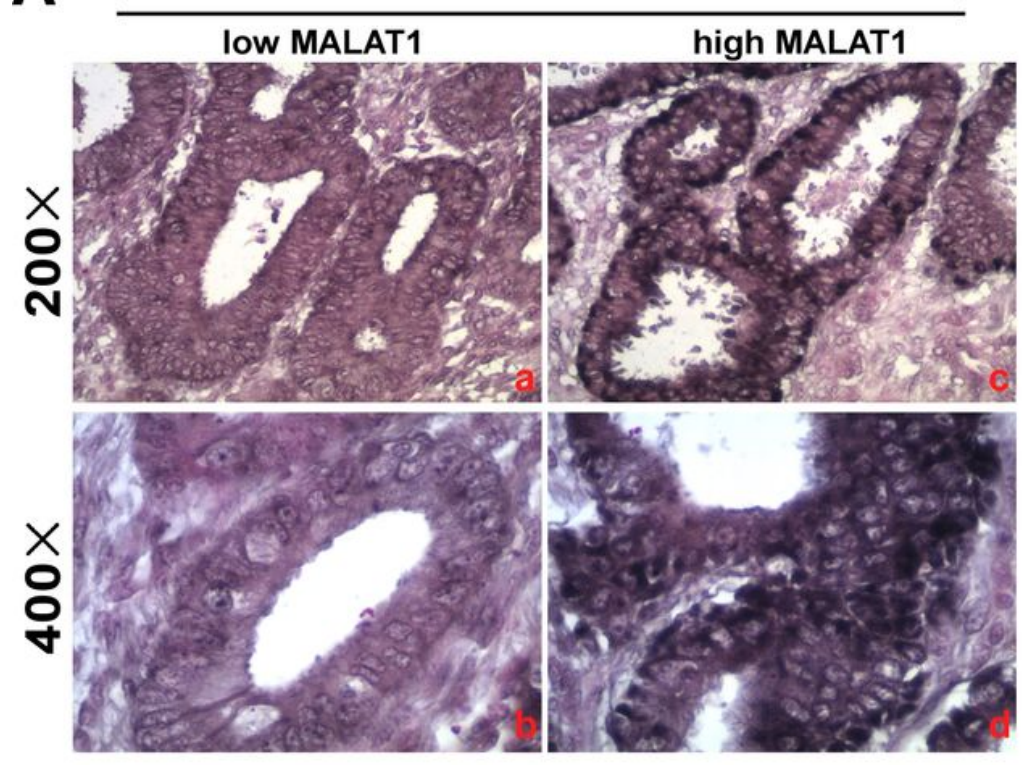

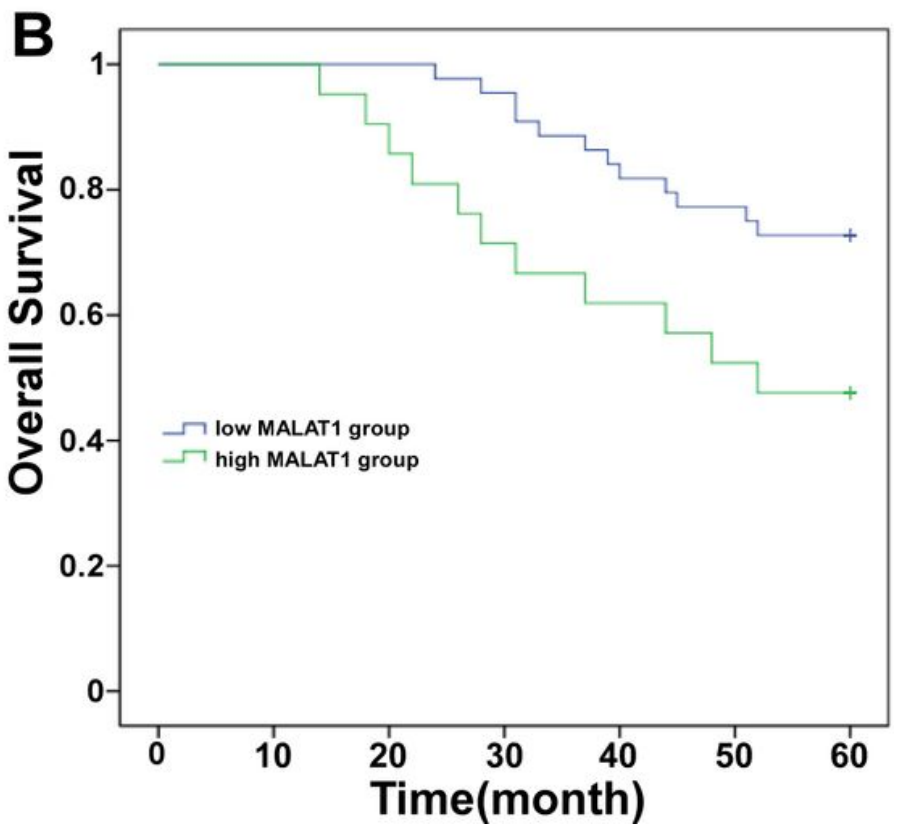

Figure 5

MALAT1 level detected in ECa tissue. (A.) ISH analysis of the MALAT1 in the 65 cases of ECa tissue. Paraffin-embedded tissue sections were stained using specific probe for MALAT1, the positive particles is purple-blue. (B.) Kaplan-Meier survival analysis of 65 EC patients, compared based on high and low MALAT1 expression groups, its expression correlates with poor outcome of ECa.
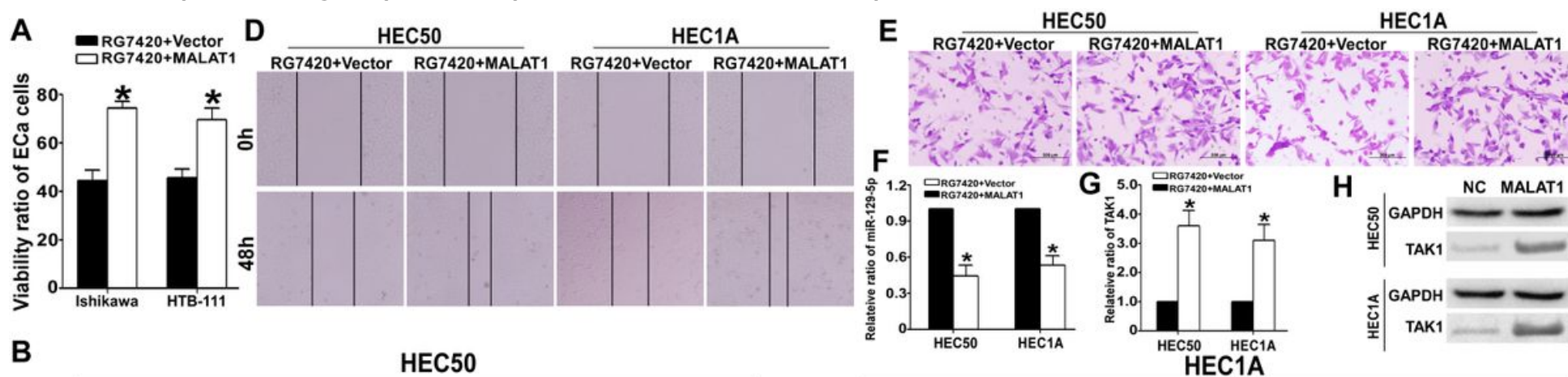

B

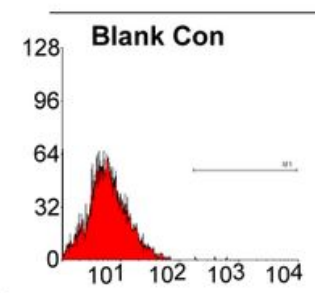

C

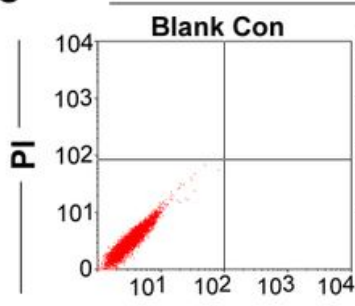

HEC50
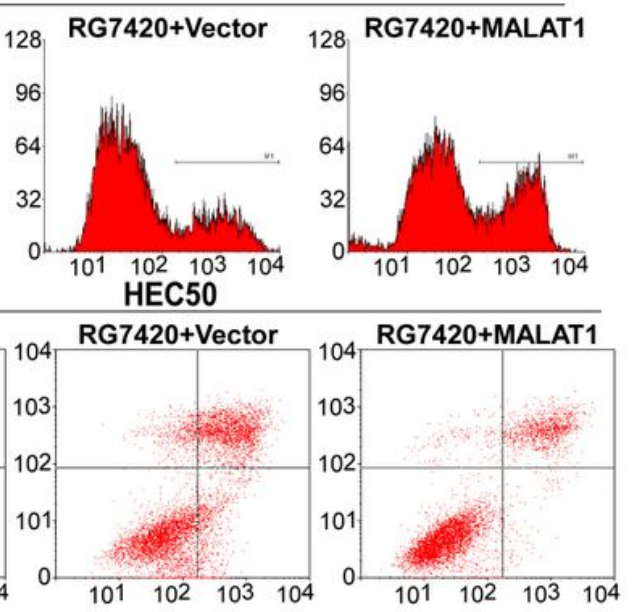
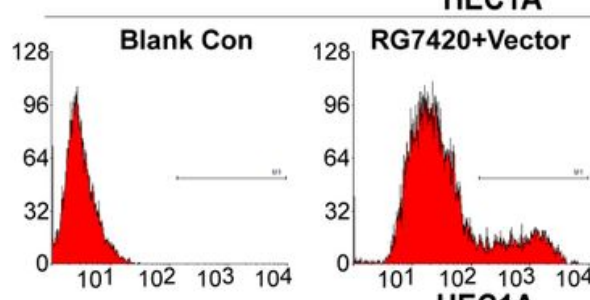

HEC1A

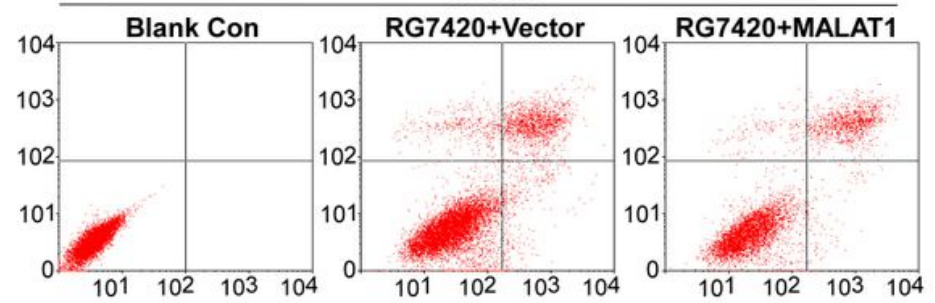

AnnexinV

Figure 6 
MALAT1 involved in RG7420 effect on ECa cells. MALAT1 decreased dramatically in ECa cells with RG7420 treatment, which referred to the proliferation and invasion of EC cells. The expression of MALAT1 was upregulated by cDNA vector in ECa cells using against RG7420 effect. (A.) Undergoing IC50 RG7420 concentration for $24 \mathrm{~h}$, the viability ratio of over-expressed MALAT1 group was remarkably higher than that in NC group by CCK8 assay ( ${ }^{*}$ means $P<0.05$ ). (B.) The DCFH-DA test displayed that over-expressed MALAT1 restrained ROS generation by RG7420. (C.) The apoptosis assay indicated that over-expressed MALAT1 inhibited the inducing apoptosis effect of RG7420. (D.) As for wound heal test, the scratched area was notly healed in the MALAT1 group than NC group. (E.) Exposing to RG7420 pressure, the transwell assay display MALAT1 signally promoted the migration than NC group. (F.) MALAT1 stile downregulated the miR-129-5p level in HEC50 and HEC1A cells by QRT-PCR. But for TAK1 level, the tendency was opposite to miR-129-5p. MALAT1 incresded the expression of TAK1 on both mRNA(G.) and $\operatorname{protein}\left(\mathrm{H}_{\text {.) }}\right.$. 